\title{
Computational Method based Laplace Adomian Decomposition for Solving Delay Differential Equations of Fractional Order
}

\author{
Zaid A. Mohammed \\ Economic and Administration College, the Iraqi University, Baghdad-Iraq. \\ Corresponding author: Zaid.amer84@yahoo.com.
}

\begin{abstract}
In this paper we present a computational method for solving delay differential equations of fractional order by employing the Laplace Adomian decomposition method. This method is combined from the Laplace transforms and the Adomian decomposition method taking into account the Caputo derivative as a motivation to describe the fractional derivative. The method is a modification of the Adomian decomposition method and is tested on two examples in order to illustrate the pertinent feature of this method the results shows that the proposed method is an effective and powerful tool for solving delay differential equations of fractional order. A comparison with the exact solution and with the existing methods such as Adomian decomposition method and homotopy analysis method is made. [DOI: $10.22401 / \mathrm{JUNS} .21 .1 .20]$
\end{abstract}

Keywords: fractional calculus, delay differential equations, Adomian decomposition method, Laplace transform.

\section{Introduction}

In recent years, the term fractional calculus has gain more attention as it could be found in many applications in physics, chemistry and engineering $[1,2]$. The fractional calculus may be defined as a generalizations of the ordinary calculus but with fractional order.

Fractional differential equations have several applications in mathematical physics, fluid flow, engineering and other areas of applications (see Miller et al., 1993; Podlubny, 1999; Jafari et al., 2007; Daftardar at al., 2007; Abdulaziz et al., 2008)[3].

The solution of fractional differential equations is one of the most important targets that mathematicians have been trying to achieve, since most of these fractional problems have no exact solution, many methods and techniques have been arisen to find analytical or numerical approaches such as spectral tau method [4], finite difference method [5], wavelet operational matrix method [6], collocation method [7, 8], finite element methods [9], spline collocation method [10] and others ([11]-[14]), [15].

Fractional delay differential equations are a very recent topic and it is a generalization of the delay differential equations to arbitrary non-integer order. Most fractional delay differential equations have no exact solutions therefore different numerical methods such as
[16-21] have been developed and applied for providing approximate solutions.

Fractional delay differential equations have found many applications in control theory, [21], agriculture [22], Chaos [23-27], and bioengineering $[28,29]$ and so on.

In this paper we shall use LaplaceAdomian decomposition method for solving non-linear delay differential equations of fractional order.

Moreover, unlike some other procedures which consider either discretization or linearization, the Laplace-Adomian decomposition method deals with the delay differential equations immediately and it is illustrates how the Laplace transform may be used to approximate the solutions of the delay differential equations of fractional order by manipulating the decomposition method.

Suheil A. Khuri [30-31] was the first to introduced the Laplace Adomian decomposition method which has been applied to evaluate analytical solutions for some fractional order differential problems such as the Bratu and Duffing equation in [33]. The Laplace Adomian decomposition method has shown a great success in terms of obtaining accurate results for nonlinear equations taking advantage of the capability of combining the two powerful techniques.

This paper is organized as follows: in section two, we recall the definition of 
fractional derivatives and fractional integration, in section three the proposed method is described, in section four two illustrative examples are given, finally we drawn a conclusions.

\section{Fractional Order Integration and Derivative:}

There are several definitions of fractional integration and derivative such as RiemannLiouville and Caputo definitions. Comparatively, the main advantage of the Caputo derivative is the capability of modelling real life phenomena that deal with fractional orders [1].

\section{Definition (1):}

The Riemann-Liouville fractional integral of order $\alpha>0$ is defined as follows:

$$
\begin{gathered}
\mathrm{I}_{\mathrm{t}}^{\alpha} \mathrm{f}(\mathrm{t})=\frac{1}{\Gamma(\alpha)} \int_{0}^{\mathrm{t}}(\mathrm{t}-\tau)^{\alpha-1} \mathrm{f}(\tau) \mathrm{d} \tau, \mathrm{t}>0, \\
\alpha \in \mathbb{R}^{+}
\end{gathered}
$$

Where $\Gamma(\alpha)$ is the Gamma function.

\section{Definition (2):}

The Caputo fractional derivative of order $\alpha>0$ is defined as follows:

$$
\begin{aligned}
& \left\{\begin{array}{cc}
{ }_{0}^{\mathrm{c}} D_{\mathrm{t}}^{\alpha} \mathrm{f}(\mathrm{t})= \\
\frac{1}{\Gamma(\mathrm{m}-\alpha)} \int_{0}^{\mathrm{t}} \frac{\mathrm{f}^{(\mathrm{m})}(\tau)}{(\mathrm{t}-\tau)^{\alpha+1-m}} \mathrm{~d} \tau & \mathrm{m}-1<\alpha<\mathrm{m} \\
\frac{\mathrm{d}^{\mathrm{m}}}{\mathrm{dx}^{\mathrm{m}}} \mathrm{f}(\mathrm{t}) & \alpha=\mathrm{m}
\end{array}\right.
\end{aligned}
$$

for $\alpha>0$, we have the following properties of the Caputo fractional derivative:

(i) ${ }_{0}^{c} D_{t}^{\alpha}\left(I_{t}^{\alpha} f(t)\right)=f(t)$.

(ii) $I_{t}^{\alpha}\left({ }_{0}^{c} D_{t}^{\alpha} f(t)\right)=f(t)-\sum_{k=0}^{n-1} f^{(k)}\left(0^{+}\right) \frac{t^{k}}{k !}$.

(iii) ${ }_{0}^{c} D_{t}^{\alpha}(C)=0, \quad C \in \mathbb{R}$.

(iv) ${ }_{0}^{c} D_{t}^{\alpha}\left(t^{\gamma}\right)=$

$$
\begin{cases}\frac{\Gamma(\gamma+1)}{\Gamma(\gamma-\alpha+1)} t^{\gamma-\alpha} \quad \gamma \in\{0,1,2,3, \cdots\}, & \gamma \geq[\alpha] \\ 0 \quad \gamma \in\{0,1,2,3, \cdots\}, & \gamma<[\alpha]\end{cases}
$$

Where $[\alpha]$ is the floor function of $\alpha$.

\section{Definition (3):}

The Laplace transform of the Caputo fractional order derivative is given by

$$
\mathcal{L}\left[{ }_{0}^{c} D_{t}^{\alpha} u(t)\right]=s^{\alpha} \mathcal{L}(u(t))-\sum_{k=0}^{n-1} s^{\alpha_{i}-k-1} u_{i}^{(k)}(0)
$$

\section{Laplace Adomian decomposition method for solving fractional order delay differential equations:}

Consider the delay differential Equations of fractional order

$$
\begin{gathered}
{ }_{0}^{c} D_{t}^{\alpha} y(t)=f(t)+N(y(t), y(\varnothing(t)), \\
n-1<\alpha<n, t>0 \ldots \ldots \ldots \ldots \ldots \ldots . . . \\
y^{(i)}(0)=y_{0}^{(i)}, i=0,1,2, \ldots, n-1 . . .
\end{gathered}
$$

Where ${ }_{0}^{c} D_{t}^{\alpha} y(t)$ is the Caputo fractional derivative of order $\alpha, \mathrm{N}$ is a nonlinear operator, $\mathrm{t}$ is the independent variable, $\varnothing(\mathrm{t})$ is the delay function, $y(\mathrm{t})$ is the unknown function and $y^{(i)}(0)$ are given constants.

In order to solve problem (1)-(2) we, apply the Laplace transformation on the both sides of equation (1), yields:

$\mathcal{L}\left({ }_{0}^{c} D_{t}^{\alpha} y(t)\right)=\mathcal{L}[f(t)+N(y(t), y(\varnothing(t))]$

Applying the linearity of the Laplace transform gives ]

$$
\mathcal{L}\left({ }_{0}^{c} D_{t}^{\alpha} y(t)\right)=\mathcal{L}(f(t))+\mathcal{L}(N(y(t), y(\varnothing(t))
$$

According to definition (3) the latter equation becomes:

$$
\begin{array}{r}
S^{\alpha} \mathcal{L}(y(t))-\sum_{k=0}^{n-1} y^{(k)}(0) S^{\alpha-k-1}= \\
F(s)+\mathcal{L}(N(y(t), y(\varnothing(t))
\end{array}
$$

Using equation (2), thus we have

$$
\begin{aligned}
& S^{\alpha} \mathcal{L}(y(t))=\sum_{k=0}^{n-1} y_{0}^{(k)} S^{\alpha-k-1}+F(s)+ \\
& \mathcal{L}(N(y(t), y(\varnothing(t))
\end{aligned}
$$

The next step in the Laplace Adomian decomposition method is to represent the unknown function $y(t)$ as an infinite series as given below

$$
y(t)=\sum_{n=0}^{\infty} y_{n}(t)
$$

And the nonlinear operator $\mathrm{N}$ is decomposed as

$$
N\left(y(t), y(\varnothing(t))=\sum_{n=0}^{\infty} A_{n}\right.
$$

Where for every $n \in N, A_{n}$ are the Adomian polynomials that can be generated for all forms of nonlinearity as $A_{n}=\frac{1}{n !} \frac{d^{n}}{d \lambda^{n}}\left[N\left(\sum_{j=0}^{\infty} \lambda^{j} y_{j}(t), \sum_{j=0}^{\infty} \lambda^{j} y_{j}(\varnothing(t))\right]_{\lambda=0}\right.$ 
Substitution equations (4) and (5) into equation (3), gives

$s^{\alpha} \mathcal{L}\left(\sum_{n=0}^{\infty} y_{n}(t)\right)=\sum_{k=0}^{n-1} y_{0}^{(k)} s^{\alpha-k-1}+$ $F(s)+£\left[\sum_{n=0}^{\infty} A_{n}\right]$

Or

$\mathcal{L}\left(\sum_{n=0}^{\infty} y_{n}(t)\right)=\frac{1}{s^{\alpha}} \sum_{k=0}^{n-1} y_{0}^{(k)} s^{\alpha-k-1}+$

$\frac{1}{s^{\alpha}} F(s)+\frac{1}{s^{\alpha}} £\left[\sum_{n=0}^{\infty} A_{n}\right]$

Comparing both sides of equ. (7), we have

$\mathcal{L}\left(y_{0}(t)\right)=\frac{1}{s^{\alpha}} \sum_{k=0}^{n-1} y_{0}^{(k)} s^{\alpha-k-1}+\frac{1}{s^{\alpha}} F(s)$

$\mathcal{L}\left(y_{n+1}\right)=\frac{1}{s^{\alpha}} £\left(A_{n}\right)$

Applying the invers Laplace transform on equations (8) and (9) we obtain, $y_{0}(t), y_{1}(t), \ldots$ recursively.

Substitution $y_{0}(t), y_{1}(t), \ldots$, into equation (4), then the solution of problem (1)-(2) is reached. The obtained series solution may converge to an exact solution if such solution exists.

\section{Illustrative Examples:}

In this section, the proposed method is used to solve the non-linear delay differential equations of fractional order and the result obtained will be compared with the existing methods and with the exact solution.

\section{Example 1:}

Consider the delay differential equation of fractional order $\alpha$,

$$
\begin{gathered}
{ }_{0}^{c} D_{t}^{\alpha} \mathrm{y}(\mathrm{t})=\mathrm{y}\left(\frac{\mathrm{t}}{3}\right)+\frac{2}{\Gamma(3-\alpha)} \mathrm{t}^{2-\alpha}-\frac{\mathrm{t}^{2}}{9}, \\
0 \leq t \leq 1,0<\alpha \leq 1
\end{gathered}
$$

With

$$
y(0)=0
$$

The exact solution of problem (10) - (11) when $\alpha=1$ is $y(t)=t^{2}$.

In the following example, the proposed method given in section three is applied as follows:

First, we apply the Laplace transform on both sides of equation (10) to obtain $\mathcal{L}\left({ }_{0}^{c} D_{t}^{\alpha} y(t)\right)=\mathcal{L}\left(y\left(\frac{t}{3}\right)\right)+\mathcal{L}\left(\frac{2}{\Gamma(3-\alpha)} t^{2-\alpha}-\frac{t^{2}}{9}\right)$
According to the definition (3) and by the linearity of the Laplace transform the above equation yields:

$$
\begin{aligned}
& S^{\alpha} \mathcal{L}(y(t)-y(0))= \\
& \quad \mathcal{L}\left(y\left(\frac{t}{3}\right)\right)+\mathcal{L}\left(\frac{2}{\Gamma(3-\alpha)} t^{2-\alpha}-\frac{t^{2}}{9}\right)
\end{aligned}
$$

Using equation (11), gives:

$\mathcal{L}(y(t))=\frac{1}{S^{\alpha}} \mathcal{L}\left(y\left(\frac{t}{3}\right)\right)+\frac{1}{S^{\alpha}}\left(\frac{2}{S^{3-\alpha}}-\frac{2}{9 S^{3}}\right)$

Therefore

$\mathcal{L}(y(t))=\frac{2}{S^{3}}-\frac{2}{9 S^{3+\alpha}}+\frac{1}{s^{\alpha}} \mathcal{L}\left(y\left(\frac{t}{3}\right)\right)$

Decomposing the solution $y(t)$ as an infinite sum, we have:

$$
\begin{aligned}
& \mathcal{L}\left(\sum_{n=0}^{\infty} y_{n}(t)\right)= \\
& \quad \frac{2}{s^{3}}-\frac{2}{9 S^{3+\alpha}}+\frac{1}{s^{\alpha}} \mathcal{L}\left(\sum_{n=0}^{\infty} y_{n}\left(\frac{t}{3}\right)\right)
\end{aligned}
$$

Comparing both sides of equ. (12), we have

$\mathcal{L}\left(y_{0}(t)\right)=\frac{2}{s^{3}}-\frac{2}{9 S^{3+\alpha}}$

And

$\mathcal{L}\left(y_{n+1}(t)\right)=\frac{1}{S^{\alpha}} \mathcal{L}\left(y_{n}\left(\frac{t}{3}\right)\right)$

Applying the invers Laplace transform on both sides of equations (13) and (14), therefore the recursive solutions are given below as:

$y_{0}(t)=t^{2}-\frac{2 t^{2+\alpha}}{9 \Gamma(3-\alpha)}$

$y_{1}(t)=\frac{2 t^{2+\alpha}}{9 \Gamma(3-\alpha)}-\frac{2 t^{2+\alpha}}{9-3^{2+\alpha \Gamma(3+2 \alpha)}}$

$y_{2}(t)=\frac{2 t^{2+2 \alpha}}{9.3^{2+\alpha \Gamma(3+2 \alpha)}}-\frac{2 t^{2+3 \alpha}}{9.3^{4+3 \alpha \Gamma(3+3 \alpha)}}$

And so on, Hence the solution of problem (10)-(11) is given by:

$y(t)=\sum_{n=0}^{\infty} y_{n}(t)$

It is clear that from the results above when $\alpha=1$ the proposed solution that we have been obtained gives us the exact solution which is $y(t)=t^{2}$.

Following Table (1) represent the approximate solution of problem (10-11) using the proposed method compared with the exact solution when $\alpha=1$ and with the Adomian decomposition method. 
Table (1)

Comparison between the approximate solution of problem (10-11) using the proposed method and Adomian decomposition method with the exact solution when $\alpha=1$

\begin{tabular}{|c||c||c||c||}
\hline \hline & $\begin{array}{c}\text { Adomian } \\
\text { decomposition } \\
\text { method } \boldsymbol{\alpha}=\mathbf{1}\end{array}$ & $\begin{array}{c}\text { The } \\
\text { proposed } \\
\text { method } \\
\boldsymbol{\alpha}=\mathbf{1}\end{array}$ & $\begin{array}{c}\text { Exact } \\
\text { solution } \\
\boldsymbol{\alpha}=\mathbf{1}\end{array}$ \\
\hline \hline 0.1 & 0.01 & 0.01 & 0.01 \\
\hline 0.2 & 0.04 & 0.04 & 0.04 \\
\hline 0.3 & 0.09 & 0.09 & 0.09 \\
\hline 0.4 & 0.16 & 0.16 & 0.16 \\
\hline 0.5 & 0.25 & 0.25 & 0.25 \\
\hline \hline 0.6 & 0.36 & 0.36 & 0.36 \\
\hline 0.7 & 0.49 & 0.49 & 0.49 \\
\hline 0.8 & 0.64 & 0.64 & 0.64 \\
\hline 0.9 & 0.81 & 0.81 & 0.81 \\
\hline \hline
\end{tabular}

Example 2:

Consider the nonlinear delay differential equations of fractional order

${ }_{0}^{c} D_{t}^{\alpha} y(t)=1-2 y^{2}\left(\frac{t}{2}\right) \quad, 0 \leq t \leq 1$,

$0<\alpha \leq 1$

With

$y(0)=0$

The exact solution of problem (15-16), when $\alpha=1$ is $y(t)=\sin t$.

The Laplace transformation is applied on equation (15) to get

$S^{\alpha} \mathcal{L}(y(t))-\sum_{k=0}^{m-1} y^{(k)}(0) S^{\alpha-k-1}=\frac{1}{s}-2 \mathcal{L}\left(y^{2}\left(\frac{t}{2}\right)\right)$

Or

$S^{\alpha} \mathcal{L}(y(t))=\sum_{k=0}^{0} y^{(k)}(0) S^{\alpha-k-1}+\frac{1}{S}-2 \mathcal{L}\left(\sum_{n=0}^{\infty} A_{n}\right)$

By the aid of equation (16) and according to the Adomians method we decomposing the solution $y(t)$ as an infinite sum, and writing the nonlinear terms as Adomians polynomials yields:

$\mathcal{L}\left(\sum_{n=0}^{\infty} y_{n}(t)=\frac{1}{s^{\alpha+1}}-\frac{2}{s^{\alpha}} \mathcal{L}\left(\sum_{n=0}^{\infty} A_{n}\right)\right.$

By comparing the both sides of equation (17), we get

$\mathcal{L}\left(y_{0}(t)\right)=\frac{1}{S^{\alpha+1}}$ and

$\mathcal{L}\left(y_{n+1}\right)=\frac{-2}{s^{\alpha}} \mathcal{L}\left(A_{n}\right)$

Applying the invers Laplace transform on both sides of equations (18) and (19), therefore the recursive solutions are given below as:

$y_{0}(t)=\frac{t^{\alpha}}{\Gamma(\alpha+1)}$

$y_{1}(t)=\frac{-2 \Gamma(\alpha+1) t^{3 \alpha}}{2^{2 \alpha} \Gamma^{2}(\alpha+1) \Gamma(3 \alpha+1)}$

$y_{2}(t)=\frac{8 \Gamma(\alpha+1) \Gamma(4 \alpha+1) t^{5 \alpha}}{\left.2^{6 \alpha^{3}}(\alpha+1) \Gamma(3 \alpha+1) \Gamma(5 \alpha+1)\right)}$

And so on. Therefore the solution of problem (15) - (16) is given by

$y(t)=\sum_{n=0}^{\infty} y_{n}(t)$

Also it is clear that form the result given in Table (2) when $\alpha=1$ the solution of the proposed method give us the exact solution which is $y(t)=\sin t$.

Following Table (2) represent a comparison between the numerical solution of problem (15)-(16) using the proposed method and the homotopy analysis method with the exact solution when $\alpha=1$.

Table (2)

Comparison between the approximate solution of problem (15)-(16) using the proposed method and homotopy analysis method with the exact solution when $\alpha=1$.

\begin{tabular}{|c||c||c|c||}
\hline $\boldsymbol{x}$ & $\begin{array}{c}\text { homotopy } \\
\text { analysis } \\
\text { method } \\
\boldsymbol{\alpha}=\mathbf{1}\end{array}$ & $\begin{array}{c}\text { The } \\
\text { proposed } \\
\text { method } \\
\boldsymbol{\alpha}=\mathbf{1}\end{array}$ & $\begin{array}{c}\text { Exact } \\
\text { solution } \\
\boldsymbol{\alpha}=\mathbf{1}\end{array}$ \\
\hline \hline 0.1 & 0.1 & 0.1 & 0.1 \\
\hline 0.2 & 0.199 & 0.199 & 0.199 \\
\hline 0.3 & 0.296 & 0.296 & 0.296 \\
\hline 0.4 & 0.389 & 0.389 & 0.389 \\
\hline 0.5 & 0.479 & 0.479 & 0.479 \\
\hline 0.6 & 0.565 & 0.565 & 0.565 \\
\hline \hline 0.7 & 0.644 & 0.644 & 0.644 \\
\hline \hline 0.8 & 0.717 & 0.717 & 0.717 \\
\hline \hline 0.9 & 0.783 & 0.783 & 0.783 \\
\hline \hline
\end{tabular}

Following Fig.(1) represent the approximate solution of problem (15-16) using Laplace Adomian decomposition method up to three 
terms for different values of $\alpha$ with a comparison with the exact solution when $\alpha=1$.

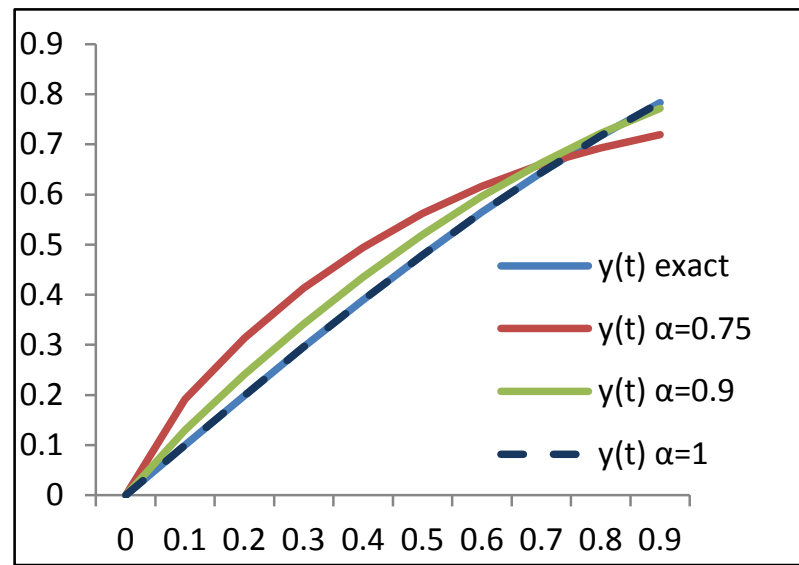

Fig.(1): Represent the approximate solution of example (2) with various values of $\alpha$ and comparing with the exact solution.

\section{Conclusions}

In this paper we introduced a simple technique by combining the Laplace transform method and Adomian decomposition method for solving delay differential equations of fractional order.

The main advantage of this method is the fact that it gives the analytical solution. The method is applied in a direct way without any discretization or linearization.

By comparing the numerical solution using the proposed method with the exact solution and that of Adomian decomposition method and homotopy analysis method, we demonstrate the accuracy and efficiency of the proposed method, and from the obtained results it may concluded that the Laplace Adomian decomposition method is a powerful method in finding the exact solution and can be used in finding the analytical solution for a wide classes of problems in fractional calculus.

\section{References}

[1]. Kilbas, A.A., Srivastava H.M. and Trujillo J.J, ELSEVIER, 2006.

[2].Podlubny, "Fractional Differential Equations", ACADEMIC press, 1999.

[3].Alshbool M.H.T., Bataineh A.S., Hashim I., Isik O. R., "Solution of fractional-order differential equations based on the operational matrices of new fractional Bernstein functions", Journal of King Saud University-Science, 2015, doi: http://dx.doi.org/10.1016/j.jksus.2015.11.00 4.

[4].E.H. Doha, A.H. Bhrawy and S.S. EzzEldien, "An efficient Legendre spectral tau matrix formulation for solving fractional subdiffusion and reaction subdiffusion equations", J.Comput. Nonlinear Dyn., 10, 021019. 8, 2015.

[5].H. Wang and N. Du, "Fast alternatingdirection finite difference methods for three dimensionalspace-fractional diffusion equations", J. Comput. Phys., 258, 305-318, 2014.

[6].M. Yi and J. Huang, "Wavelet operational matrix method for solving fractional differential equations with variable coefficients", Appl. Math. Comput., 230, 383-394, 2014.

[7].A.H. Bhrawy, E.H. Doha, S.S. Ezz-Eldien and R.A.V. Gorder, "A new Jacobi spectral collocation method for solving $1+1$ fractional Schrödinger equations and fractional coupled Schr"odinger systems", Eur. Phys. J. Plus, 129 (12), 1-21, 2014.

[8].M.A. Abdelkawy, S.S. Ezz-Eldien and A.Z.M. Amin, "Jacobi spectral collocation scheme for solving Abel's integral equations", Progr. Fract. Differ. Appl., 1, 187-200, 2015.

[9].P. Zhuang, F. Liu, I. Turner and Y.T. Gu, "Finite volume and finite element methods for solving a one-dimensional spacefractional Boussinesq equation", Appl. Math. Model., 38, 3860-3870, 2014.

[10]. X. Yang, H. Zhang and D. Xu, "Orthogonal spline collocation method for the two dimensional fractional subdiffusion equation", J. Comput. Phys., 256, 824-837, 2014.

[11]. R.M. Hafez, S.S. Ezz-Eldien, A.H. Bhrawy, E.A. Ahmed and D. Baleanu, A Jacobi Gauss-Lobatto and Gauss-Radau collocation algorithm for solving fractional Fokker-Planck equations, Nonlinear Dyn., 82 (3), 1431-1440, 2015.

[12]. A.H. Bhrawy, M.A. Abdelkawy and S.S. Ezz-Eldien, Efficient spectral collocation algorithm for a two-sided space fractional Boussinesq equation with non-local conditions, Mediterr. J. Math., 2015, DOI:10.1007/s00009-015-0635-y. 
[13]. Song L. and Wang W. "A new improved Adomian decomposition method and its application to fractional differential equations", Appl. Math. Model., 37, 1590$1598 \mathrm{~m} 2013$.

[14]. Wang L., Ma Y. and Meng Z., "Haar wavelet method for solving fractional partial differential equations numerically", Appl. Math. Comput., 227, 66-76, 2014.

[15]. Ezz-Eldien S.S., "New quadrature approach based on operational matrix for solving a class of fractional variational problems", J. Comput. Phys. 2016, http://dx.doi.org/10.1016/j.jcp.2016.04.045.

[16]. Wang Z., "A numerical method for delayed fractional-order differential equations", J. Appl. Math. 2013 7, doi:10.1155/2013/256071. Article ID 256071.

[17]. Moghaddam B.P., Mostaghim Z.S., "A numerical method based on finite difference for solving fractional delay di_erential equations", J. Taibah Univ. Sci.7, 120127, 2013.

[18]. Morgado M.L., Ford N.J., Lima P.M., "Analysis and numerical methods for fractional differential equations with delay", J. Comput. Appl. Math. 252, 159168, 2013.

[19]. Mohammed O.H., Wadi Q., "A modified method for solving delay differential equations of fractional order", IOSR journal of mathematics (IOSR-JM), 12(3), Ver.VII, 15-21, May-June 2016.

[20]. Mohammed O.H., Khlaif A.I., "Adomian decomposition method for solving delay differential equations of fractional order", IOSR journal of mathematics (IOSR-JM), 10 (6), Ver.I, 01-05 Nov.-Dec. 2014.

[21]. Mohammed O.H., Khlaif A.I., "homotopy analysis method for solving delay differential equations of fractional order", mathematical theory and modeling, (14), 2014.

[22]. Monje C A, Y Q Chen, B M Vinagre, D $\mathrm{Y}$ Xue and $\mathrm{V}$ Feliu, Fractional-order systems and controls: Fundamentals and applications (Springer-Verlag, London, 2010).

[23]. Feliu V, R Rivas and F J Castillo, "Fractional robust control to delay changes in main irrigation canals", Proceedings of the $16^{\text {th }}$ International Federation of
Automatic Control World Congress (Prague, Czech Republic, 2005).

[24]. Wang D and Yu J., Electronic Sci. Tech. of China 6(3), 225, 2008.

[25]. Lu J G, Chin. Phys. 15(2), 301, 2006.

[26]. Bhalekar S and V Daftardar-Gejji, Commun. Nonlinear Sci. Numer. Simul. 15 (8), 2178, 2010.

[27]. Daftardar-Gejji V, Bhalekar S. and P Gade, Pramana J. Phys. 79(1), 61, 2012.

[28]. Bhalekar S, Signals, Image and Video Processing 6(3), 513, 2012.

[29]. Bhalekar S, V Daftardar-Gejji, D Baleanuand R Magin, Comput. Math. Appl.61, 1355, 2011.

[30]. Bhalekar S,V Daftardar-Gejji, D Baleanu and R Magin, Int. J. Bifurcation Chaos 22(4), 1250071, 2012, DOI: 10.1142/S021812741250071X.

[31]. Khuri, S.A., J. Math. Annl. Appl., 4, 141, 2001.

[32]. Khuri, S.A., Appl. Math. Comp., 147, 31, 2004.

[33]. Syam, M.I., Hamdan, A., Appl. Math. Comp., 176, 704, 2006.

[34]. Yusufoğlu (Agadjanov), E., Appl. Math. Comp., 177, 572, 2006.

[35]. Wazwaz, A.M., Mehanna, M.S., Inter. J. of Nonlinear Science, 10, 248, 2010.

[36]. Hendi, F.A., Studies in Nonlinear Sciences, 2, 129, 2011.

[37]. Khan, M., Hussain, M., Numer. Algor, 56, 211, 2011.

[38]. Ahmad J., Bibi Z., Noor K., "Laplace Decomposition Method Using He's Polynomial to Burgers Equation", Journal of Science and Arts, Year 14, No. 2(27), pp. 131-138, 2014. 